\title{
Spin-orbit interaction in InAs/GaSb heterostructures quantified by weak antilocalization
}

\author{
F. Herling, ${ }^{1,2}$ C. Morrison, ${ }^{1,3}$ C. S. Knox,${ }^{1,4}$ S. Zhang,,${ }^{5}$ O. Newell, ${ }^{3}$ M. Myronov, ${ }^{3}$ E. H. Linfield, ${ }^{4}$ and C. H. Marrows ${ }^{1}$ \\ ${ }^{1}$ School of Physics and Astronomy, University of Leeds, Leeds, LS2 9TJ, United Kingdom \\ ${ }^{2}$ Novel Materials Group, Institut für Physik, Humboldt-Universität zu Berlin, 12489 Berlin, Germany \\ ${ }^{3}$ Department of Physics, University of Warwick, Coventry, CV4 7AL, United Kingdom \\ ${ }^{4}$ School of Electronic and Electrical Engineering, University of Leeds, Leeds, LS2 9TJ, United Kingdom \\ ${ }^{5}$ EPSRC National Centre for III-V Technologies, Department of Electronic and Electrical Engineering, University of Sheffield, \\ Sheffield S1 4DE, United Kingdom
}

(Received 1 November 2016; revised manuscript received 6 March 2017; published 10 April 2017)

\begin{abstract}
We study the spin-orbit interaction (SOI) in InAs/GaSb and InAs quantum wells. We show through temperatureand gate-dependent magnetotransport measurements of weak antilocalization that the dominant spin-orbit relaxation mechanism in our low-mobility heterostructures is Elliott-Yafet and not Dyakonov-Perel in the form of the Rashba or Dresselhaus SOI as previously suggested. We compare our findings with recent work on this material system and show that the SOI length lies within the same range. The SOI length may be controlled using an electrostatic gate, opening up prospects for developing spintronic applications.
\end{abstract}

DOI: 10.1103/PhysRevB.95.155307

\section{INTRODUCTION}

InAs/GaSb heterostructures have seen renewed research interest in recent years. Early work focused on coupling a two-dimensional electron gas (2DEG) in the InAs layer with a 2D hole gas in the GaSb layer, creating hybridized electronhole quantum states [1-8]. Much later, it was predicted that a topologically insulating state could be created in an InAs/GaSb heterostructure [9] by using band inversion due to the band-edge positions of the conduction and valence bands in InAs and GaSb, respectively, to create an energy gap in the bulk and counterpropagating spin-polarized helical edge states, known as the quantum spin-Hall effect (QSHE). Shortly after this theoretical prediction the bulk topological gap was demonstrated experimentally [10], and recent research has focused on demonstrating spin-polarized edge transport and confirmation of the existence of the QSHE [11-20].

The Rashba spin-orbit interaction (SOI) has been studied in 2DEGs in several InAs and InGaAs quantum-well (QW) heterostructures [21-28]. The SOI gives rise to the conventional spin-Hall effect (SHE), which is useful as a means of manipulating spin in a spintronic device, while its inverse can be used for detecting spin-polarized currents such as those in the helical edge states generated by the QSHE. The use of the SHE to detect such helical edge states in this manner was recently achieved in an HgTe-based topological insulator [29], and a similar experimental approach could be employed in the InAs/GaSb system. One of the underlying causes of spin relaxation is the Dyakonov-Perel (DP) mechanism, which originates in the spatial inversion asymmetry of the heterostructure [30]. Another contribution is momentum scattering from phonons and impurities, known as the Elliot-Yafet (EY) mechanism, owing to the mixing of spin-up and spin-down states by the SOI of the lattice ions [31]. It is important to understand the effect of the proximity of the QWs to each other, and any induced interfacial effects, on the strength of the SOI and the relative contributions of momentum scattering (EY) and the SOIs (DP) arising from inversion asymmetry (bulk and structural).
Here we show, through magnetotransport weak antilocalization (WAL) measurements of the temperature and gate dependence of the relevant scattering length scales, that the dominant underlying spin-relaxation mechanism in our InAs/GaSb heterostructure is EY, and not DP in the form of the Rashba SOI owing to structural inversion symmetry, as previously suggested [22-24,26]. We do this by demonstrating that the length for spin relaxation may be tuned using an electrostatic gate controlling the mean-free path. Finally, we report our results obtained from an InAs QW, which also shows strong indicators for the EY mechanism.

\section{EXPERIMENTAL SETUP}

We grew InAs/GaSb heterostructures using solid-source molecular-beam epitaxy on (100) GaAs substrates, with varying thicknesses of the InAs and GaSb layer and varying AlSb barriers. A buffer of AlSb/GaAlSb was used to relax lattice mismatch strain and provide a pseudosubstrate for growth of the electrically active QW layers. The structures are capped with GaSb to prevent oxidation. The layer structures of the two heterostructures studied here are shown in Fig. 1; they are labeled A and B and are thus referred to throughout.

Magnetotransport measurements were performed in the temperature range $0.3-1.5 \mathrm{~K}$ using an Oxford Instruments $\mathrm{He}$ liox $\mathrm{AC}-\mathrm{V}{ }^{3} \mathrm{He}$ system with a $12-\mathrm{T}$ superconducting magnet, and at temperatures above $1.5 \mathrm{~K}$ an Oxford Instruments ${ }^{4} \mathrm{He}$ flow cryostat with an 8-T superconducting magnet was used. In all measurements, the field was applied perpendicular to the plane of the device. Measurements were performed using a 50- $\mu \mathrm{m}$ wide and $250-\mu \mathrm{m}$ long Hall bar geometry, fabricated by optical lithography, using a wet-etching process to define the mesa, and 100-nm thick AuGeNi to form contacts that are Ohmic at all temperatures regardless of prior annealing. The top gate stack comprises a $30-\mathrm{nm}$ thick layer of $\mathrm{Al}_{2} \mathrm{O}_{3}$ with a 100 -nm thick $\mathrm{Cr} / \mathrm{Au}$ electrode. The back gate was fabricated by depositing and annealing AuGeNi on the highly doped substrate. An optical microscope image of a typical device is shown in an inset to Fig. 2. In addition, square and Greek cross 
(a)

\begin{tabular}{|c|}
\hline $3 \mathrm{~nm} \mathrm{GaSb}$ \\
\hline $50 \mathrm{~nm} \mathrm{AISb}$ \\
\hline $8 \mathrm{~nm} \mathrm{GaSb}$ \\
\hline $15 \mathrm{~nm}$ InAs \\
\hline $50 \mathrm{~nm} \mathrm{AISb}$ \\
\hline $1.2 \mu \mathrm{m} \mathrm{Al}{ }_{0.8} \mathrm{Ga}_{0.2} \mathrm{Sb}$ \\
\hline $100 \mathrm{~nm}$ AlSb \\
\hline $200 \mathrm{~nm}$ GaAs \\
\hline GaAs (100) substrate \\
\hline
\end{tabular}

(b)

\begin{tabular}{|c|}
\hline $3 \mathrm{~nm} \mathrm{GaSb}$ \\
\hline $50 \mathrm{~nm}$ AlSb \\
\hline $15 \mathrm{~nm} \mathrm{InAs}$ \\
\hline $50 \mathrm{~nm}$ AlSb \\
\hline $1.2 \mu \mathrm{m} \mathrm{Al} \mathrm{ol}_{.8} \mathrm{Ga}_{0.2} \mathrm{Sb}$ \\
\hline $100 \mathrm{~nm}$ AlSb \\
\hline $200 \mathrm{~nm}$ GaAs \\
\hline GaAs (100) substrate \\
\hline
\end{tabular}

$n$ in $10^{11} \mathrm{~cm}^{-2}$

$(19 \pm 1)$

$\mu$ in $\mathrm{cm}^{2} / \mathrm{Vs}$

$(2,900 \pm 100)$

$l_{S O}$ in $\mathrm{nm}$

$(150 \pm 8)$

$(82 \pm 6)$

$(280 \pm 10)$
$(7.4 \pm 0.1)$

$(10,300 \pm 100)$

$(380 \pm 30)$

$(150 \pm 20)$

$(950 \pm 50)$

FIG. 1. Comparison of the two wafers studied. The substrates are $n$-doped; the InAs/GaSb wafer A has an interface doping with $\mathrm{Si}$ atoms of $10^{11} \mathrm{~cm}^{-2}$. The charge-carrier densities, mobilities, and characteristic lengths are given at $1.7 \mathrm{~K}$.

van der Pauw geometries were used. Electrical measurements were performed using an ac current excitation of $1 \mu \mathrm{A}$ or 100 $\mathrm{nA}$ at $119.77 \mathrm{~Hz}$ and voltages were measured using Stanford Research Systems model 830 lock-in amplifiers.

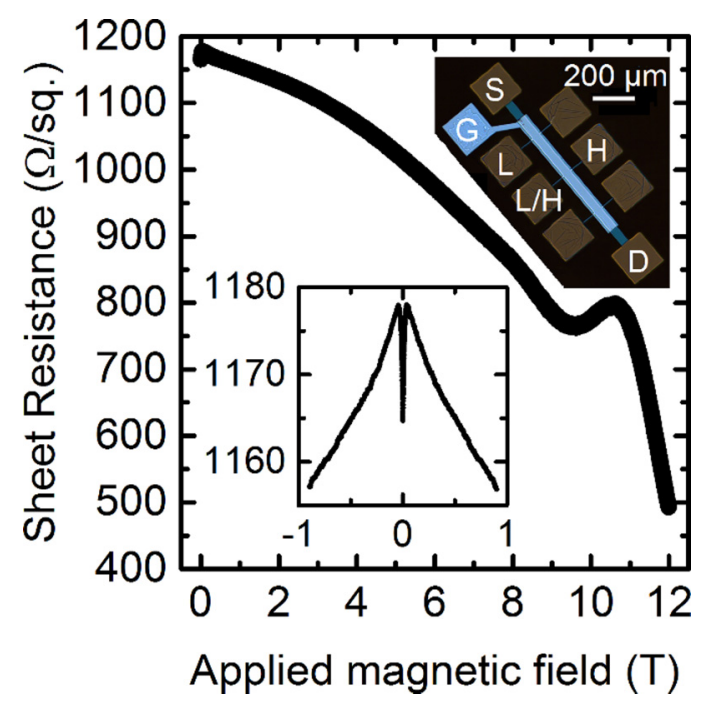

FIG. 2. Magnetoresistance of an InAs/GaSb QW Hall bar sample (wafer A) at $340 \mathrm{mK}$ and applied magnetic field of up to $12 \mathrm{~T}$. Inset (top): Optical micrograph of the gated Hall bar geometry used here with labels for contacts $(\mathrm{S}=$ source, $\mathrm{D}=$ drain, $\mathrm{G}=$ gate, $\mathrm{L}=$ longitudinal voltage, $\mathrm{H}=$ Hall voltage). Inset (bottom): Low-field magnetoresistance highlighting the WAL dip at zero field.

\section{RESULTS}

Carrier densities and mobilities at $1.7 \mathrm{~K}$ are given in Fig. 1 for both wafers. The carrier density was calculated from the Hall voltage, which was fully linear for low fields and displays plateaus for high fields in accordance with the Shubnikov-de Haas ( $\mathrm{SdH}$ ) oscillations. The majority carriers are electrons, determined from the sign of the Hall coefficient. There was no evidence of band inversion for top-, back-, or double-gated devices, perhaps due to the high carrier density and low mobility, so we cannot contribute to present arguments regarding recent findings for SOI close to the charge-neutrality point [32].

Both wafers A and B exhibit WAL. Figure 2 depicts the magnetoresistance for wafer $\mathrm{A}$, in which there is a $\mathrm{GaSb}$ layer next to the InAs QW between the AlSb barriers. At low fields the behavior shows the characteristic dip of WAL, which may be seen more clearly in the inset, with transitions to weak localization and negative magnetoresistance above $500 \mathrm{mT}$, which suggests that a high density of impurities is present [30]. This would also explain the low mobility and high carrier density across all devices fabricated from this wafer. Other reasons for the unusually low mobility could be lattice mismatch or other interface effects.

Angular-dependent measurements showed that the WAL and the Hall voltage are a sinusoidal function of the angle and vanish for an in-plane magnetic field, which demonstrates the two-dimensional nature of the carrier confinement within the QW.

The magnetoconductivity for all devices for different temperatures and applied gate voltages was fitted with the Hikami-Larkin-Nagaoka (HLN) model [33], which gives us three fitting parameters as characteristic lengths: the SOI length $l_{S O}$, which gives the average distance traveled by an electron before a flip in spin occurs, the mean-free path $l_{e}$ between elastic scattering events, and the phase-coherence length $l_{\phi}$ between inelastic scattering events. An example fit is shown in Fig. 3. The fit matches the experimental data points for the low-field range and results are independent of the chosen interval as long as it stays in the area in which WAL is present [below $400 \mathrm{mT}$ (A) or $200 \mathrm{mT}$ (B)]. Beyond that it deviates owing to the negative high-field magnetoresistance.

An advantage of low-mobility samples lies in the distinct WAL of the magnetoresistance, which can be more precisely fitted to obtain characteristic lengths. Accordingly, wafer A shows smaller errors for the characteristic lengths than wafer B, which has a higher mobility and lower carrier density (see Fig. 1). A disadvantage is the damping of the Shubnikov-de Haas oscillations for high fields, so that only one oscillation (or two for wafer B) is visible and we cannot report high-field SOI values through measurements of beating in the $\mathrm{SdH}$ oscillations, another common magnetotransport technique [22,24,26,34]. However, for certain materials the values for SOI extracted at low field from WAL are more accurate than the values deduced from highfield beating in the $\mathrm{SdH}$ oscillations as the latter include the Zeeman effect [25] or intersubband scattering [35] which causes additional uncertainty in the fast Fourier transform method used to determine the spin-split carrier densities [36]. 


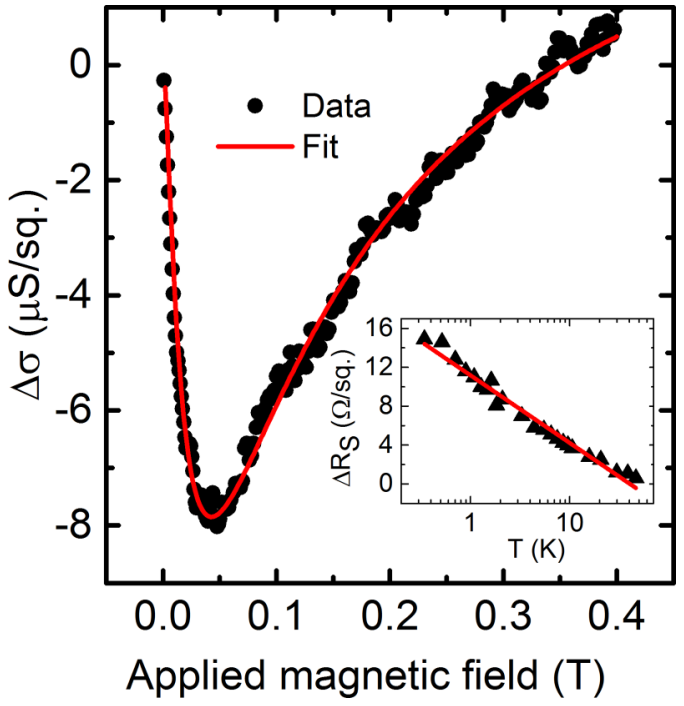

FIG. 3. Change in conductivity for an InAs/GaSb QW Hall bar sample (wafer $\mathrm{A}$ ) at $1.7 \mathrm{~K}$ as a function of perpendicular magnetic field. The black data points show the measured values; the red solid line shows the HLN model fit with the following fit parameters: $l_{S O}=147 \mathrm{~nm}, l_{e}=77 \mathrm{~nm}$, and $l_{\varphi}=289 \mathrm{~nm}$. The fit and data match for the pictured low-field range; for higher fields the background negative magnetoresistance leads to deviation from this behavior and is excluded from the fit. Inset: The absolute change in sheet resistance for low fields as a function of temperature on a log scale. The black data points show the measured values; the red solid line shows a linear fit.

\section{A. Temperature dependence}

The change in resistance due to WAL, i.e., the quantum correction to the conduction at low temperatures for low fields, shows a logarithmic temperature dependence (Fig. 3 inset) which is the expected dependence for WAL due to the powerlaw behavior of the lifetimes (spin/elastic/inelastic scattering), which can be converted to the lengths reported here [37].

The temperature dependence of the three characteristic lengths for wafer A is shown in Fig. 4. For low temperatures (up to about $5 \mathrm{~K}$ ), a $T^{-1 / 2}$ behavior is seen in the phase-coherence length, with a linear increase in the mean-free path and SOI length. For higher temperatures (above $5 \mathrm{~K}$ ) the latter two have a very weak temperature dependence and the phase-coherence length decreases linearly with increasing temperature. This suggests that electron-electron interactions are the dominant inelastic scattering mechanism for temperatures below $\sim 5 \mathrm{~K}$ and that the electron-phonon interaction dominates at temperatures above that value [38]. The temperature independence of the elastic and SOI lengths suggests that these scattering rates are dominated by impurities, which corresponds to the analysis of the magnetoresistance data and suggests that the EY mechanism dominates in this heterostructure. The exponential drop in mean-free path for the lowest temperatures (below about $1 \mathrm{~K}$ ) is paralleled by the SOI length as expected for our conclusion but cannot be explained, as, for example, a freezing out of the impurities would show an opposite trend.

The inset in Fig. 6 shows the equivalent temperature dependence for of the SOI length for wafer B. It follows the

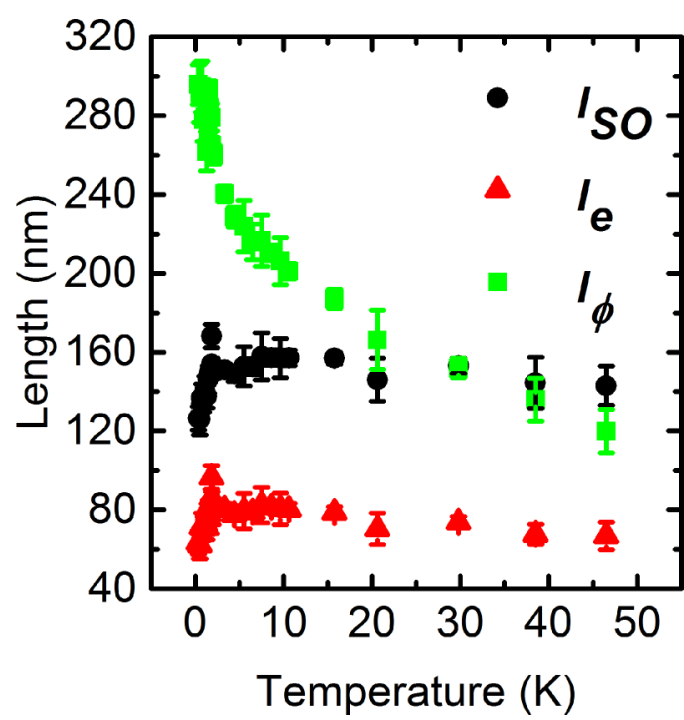

FIG. 4. Characteristic lengths from the HLN fit as a function of temperature for the InAs/GaSb wafer (A). In agreement with the model, WAL only occurs for temperatures where $l_{\varphi}$ is of the order of $l_{S O}$ or around $(\sim 50 \mathrm{~K}$; see also the inset in Fig. 3).

same behavior in the temperature range from 1.6 to $12 \mathrm{~K}$ as wafer A for higher temperatures. The characteristic lengths for both wafers at $1.7 \mathrm{~K}$ are listed in Fig. 1. In accordance with our interpretation of the magnetoresistance and the temperature behavior, a weaker SOI is seen for the lower carrier-density wafer (B), which is the expected trend for the EY mechanism, but could also be explained by the DP mechanism, which originates in the missing bulk-inversion symmetry [30]. The longer elastic and inelastic scattering lengths coincide with the high carrier mobility and can be explained with a lower impurity concentration, which would also lead to a shorter SOI length in the EY mechanism.

\section{B. Gate dependence}

With the double- (top and back) gated Hall bar samples for wafer A, the electrical transport properties (Hall sheet carrier density and mean-free path) could be varied by around $10 \%$. The bottom inset in Fig. 5 shows that as the external electric field is made more negative the SOI length becomes shorter due to a change in mobility. We interpret this negative dependence as another reason to disregard Dresselhaus SOI as a dominant factor in our samples, because the SOI length would then be independent of changes in the local electric field. This is consistent with single InAs QWs, where the Dresselhaus term was responsible for less than 5\% of the SOI [39]. The Rashba SOI length (structural or interface inversion symmetry) is theoretically predicted to be also controllable by a gate voltage, but previous experiments for InAs QWs did not provide a definite experimental confirmation of the sign of the correlation. They either did not vary carrier density [22] or did so by illumination with a light-emitting diode, rather than applying a gate voltage [23]. Experiments with gate voltages either reported no gate dependence [24] or a negative dependence for top-gate voltages [26] as in our samples. Most recently $\mathrm{Kim}$ et al. found that the dependence is determined 


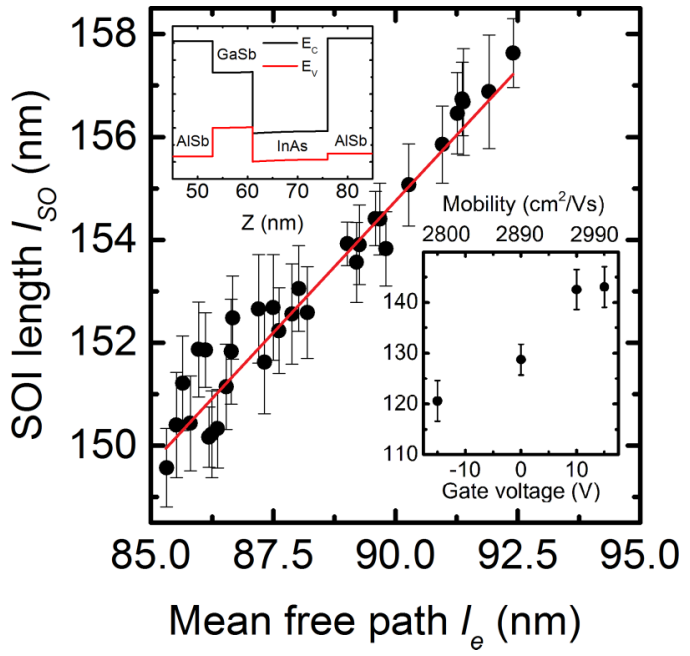

FIG. 5. SOI length as a function of the mean-free path controlled by an applied top- and back-gate voltage for wafer A at $1.7 \mathrm{~K}$. Inset (top): Calculated band diagram showing the lowest conduction and the highest valence band energy; the band inversion inside the QW is also clearly evident. The Fermi level lies between the two band edges and only the first subband is populated. Inset (bottom): SOI length as a function of the Hall mobility controlled by an applied top-gate voltage for wafer A at $340 \mathrm{mK}$.

by the QW potential gradient in accordance with theoretical predictions $[21,28,40]$. So, the negative dependence we report here could be caused by DP with Rashba SOI or EY.

\section{DISCUSSION}

The top inset in Fig. 5 shows the calculated band diagram of the InAs/GaSb heterostructure of wafer A. We performed the calculation with nextnano ${ }^{3}$, a three-dimensional Poisson and Schrödinger solver [41]. There is only a small positive difference in energy across the InAs layer of $\sim 30 \mathrm{meV}$. It is unlikely that such a small gradient would cause Rashba SOI of the order we report here, and furthermore it would lead to a negative dependence of the SOI length on a top-gate voltage. It would also lead to constant mobility [42], but we see a linear mobility dependence on gate voltage, most likely caused by scattering from impurities. We therefore conclude that the SOI length is varied due to changes in the mean-free path tuned by an external gate voltage.

In Fig. 5, a linear dependence of $l_{S O}$ on $l_{e}$ is seen for wafer A as the gate voltage is varied, just as we would expect for the EY mechanism, because spin relaxation is induced by scattering. Therefore, the DP mechanism (arising from Dresselhaus and Rashba SOI) seems to be negligible, as this would show a dependence of $l_{S O} \sim l_{e}^{-1}$, because spin precession is restarted by scattering $[30,43]$.

To compare our reported SOI length value with the Rashba parameter $\alpha$ from other experiments with InAs QWs, we used

$$
\alpha=\frac{\hbar^{2}}{m^{*} l_{S O}}
$$

to calculate the SOI length for different sources. We note that there is a variation in the reported accompanying effective
TABLE I. Comparison of the reported minimal SOI lengths derived from the largest spin-splitting parameter $\alpha$ reported, calculated with the accompanying effective mass $m^{*}$ or the value taken from literature ( $m^{*}=0.023$ electron mass).

\begin{tabular}{lcc}
\hline \hline Author & Min. $l_{S O}(\mathrm{~nm})$ & $m^{*}$ \\
\hline Luo et al. [22] & 154 & 0.055 \\
Heida et al. [24] & 317 & 0.040 \\
Grundler [26] & 47 & 0.036 \\
Schierholz et al. [27] & 167 & 0.026 \\
Kim et al. (2014) [28] & 221 & 0.050 \\
Kim et al. (2013) [21] & 237 & \\
Park et al. [39] & 473 & \\
InAs/GaSb (this work) & 150 & \\
InAs (this work) & 380 & \\
\hline \hline
\end{tabular}

mass $m^{*}$. The results are presented in Table I and show that SOI length due to the EY mechanism that we measure here is in the range of the reported Rashba SOI lengths and cannot be neglected in a thorough investigation of spin-relaxation mechanisms.

Figure 6 shows the $l_{S O}-l_{e}$ curve for wafer $\mathrm{B}$ as the gate voltage is varied. The linear trend we see for our InAs/GaSb is repeated, which points to the EY mechanism. Our calculations show an even smaller potential difference inside the QW of $10 \mathrm{meV}$, which of course could be affected by a high level of impurities. In contrast to wafer A we do not see linear dependence of mobility and mean-free path on gate voltage. An explanation could be the larger variation of the transport parameters by a higher gate voltage owing to a more stable back gate. Therefore $l_{e}$ is varied over a larger range and could show dependence outside of the narrow picture of Fig. 5. We

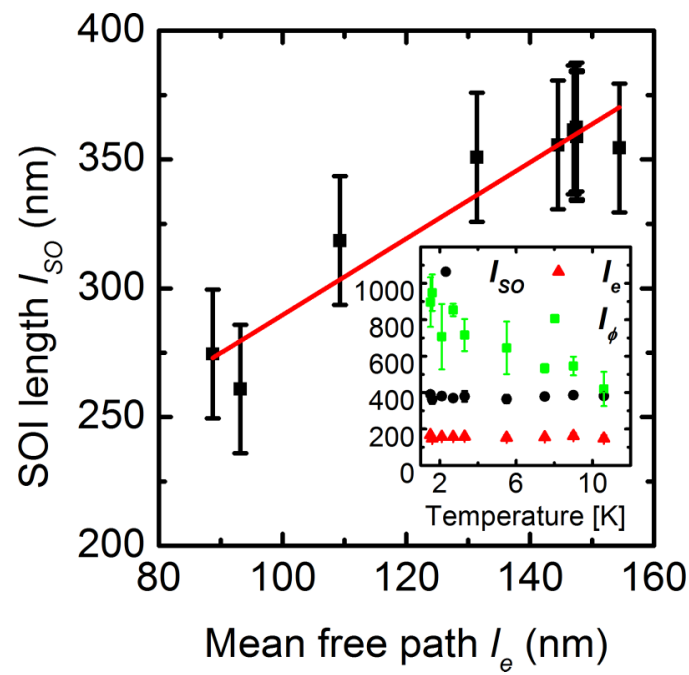

FIG. 6. $l_{S O}-l_{e}$ graph for the InAs wafer (B) at $1.7 \mathrm{~K}$. Only a back gate was used to vary the transport parameters. Inset: The temperature dependence of the three characteristic lengths obtained from the same wafer matches the InAs/GaSb wafer (A) in the higher temperature range. The phase-coherence length for wafer $\mathrm{B}$ is linear in temperature from 1.7 up to $\sim 15 \mathrm{~K}$, where it falls below the SOI length (which is, as well as $l_{e}$, almost temperature independent) and consequently no WAL is observable. 
conclude that it is reasonable to say that the EY mechanism plays an important role in our InAs heterostructures but further research is necessary.

\section{CONCLUSION}

In conclusion, we report measurements of the spin-orbit interaction in InAs/GaSb and InAs quantum wells using weak antilocalization. We conclude that the dominant spinrelaxation mechanism, at least in the InAs/GaSb heterostructure reported here, is the Elliott-Yafet mechanism, which also contributes in our InAs sample. We dismiss inversion asymmetry effects (Dyakonov-Perel) which have been used as an explanation previously and report that the SOI length is controllable by a gate voltage which makes its application in spintronic devices possible. We also add to the ongoing discussion about gate-controllable Rashba SOI by showing that a thorough experimental procedure has yet to be reported. To account for all spin-relaxation mechanisms the dependence of the SOI parameter on the mean-free path, the gate voltage, and the intrinsic carrier density have to be considered.

\section{ACKNOWLEDGMENTS}

We acknowledge helpful discussions with S. F. Fischer and O. Chiatti. We also thank M. Rosamond for advice in all cleanroom work, J. Batley for assistance with low-temperature measurements, and G. Burnell for software support. This work was supported by the Engineering and Physical Sciences Research Council, the EPSRC platform grant "Spintronics at Leeds" (Grant No. EP/M000923/1), the EPSRC National Centre for III-V Technologies, and the Erasmus+ program (F.H.).

The data associated with this paper are publicly available from the University of Leeds Data Repository at the following URL: https://doi.org/10.5518/180.
[1] M. Drndic, M. P. Grimshaw, L. J. Cooper, D. A. Ritchie, and N. K. Patel, Appl. Phys. Lett. 70, 481 (1997).

[2] R. J. Wagner, B. V. Shanabrook, M. J. Yang, and J. R. Waterman, Superlattices Microstruct. 21, 95 (1997).

[3] M. J. Yang, C. H. Yang, B. R. Bennett, and B. V. Shanabrook, Phys. Rev. Lett. 78, 4613 (1997).

[4] T. P. Marlow, L. J. Cooper, D. D. Arnone, N. K. Patel, D. M. Whittaker, E. H. Linfield, D. A. Ritchie, and M. Pepper, Phys. Rev. Lett. 82, 2362 (1999).

[5] Y. Vasilyev, S. Suchalkin, K. von Klitzing, B. Meltser, S. Ivanov, and P. Kop'ev, Phys. Rev. B 60, 10636 (1999).

[6] K. Suzuki, S. Miyashita, and Y. Hirayama, Phys. Rev. B 67, 195319 (2003).

[7] C. Petchsingh, R. J. Nicholas, K. Takashina, N. J. Mason, and J. Zeman, Phys. Rev. B 70, 155306 (2004).

[8] K. Nilsson, A. Zakharova, I. Lapushkin, S. T. Yen, and K. A. Chao, Phys. Rev. B 74, 075308 (2006).

[9] C. Liu, T. L. Hughes, X. L. Qi, K. Wang, and S. C. Zhang, Phys. Rev. Lett. 100, 236601 (2008).

[10] I. Knez, R. R. Du, and G. Sullivan, Phys. Rev. B 81, 201301 (2010).

[11] I. Knez, R. R. Du, and G. Sullivan, Phys Rev. Lett. 107, 136603 (2011).

[12] I. Knez, R. R. Du, and G. Sullivan, Phys. Rev. Lett. 109, 186603 (2012).

[13] I. Knez, C. T. Rettner, S.-H. Yang, S. S. P. Parkin, L. Du, R.-R. Du, and G. Sullivan, Phys. Rev. Lett. 112, 026602 (2014).

[14] C. Charpentier, S. Fält, C. Reichl, F. Nichele, A. Nath Pal, P. Pietsch, T. Ihn, K. Ensslin, and W. Wegscheider, Appl. Phys. Lett. 103, 112102 (2013).

[15] A. Nichele, N. Pal, P. Pietsch, T. Ihn, K. Ensslin, C. Charpentier, and W. Wegscheider, Phys. Rev. Lett. 112, 036802 (2014).

[16] E. M. Spanton, K. C. Nowack, L. Du, G. Sullivan, R. R. Du, and K. A. Moler, Phys. Rev. Lett. 113, 026804 (2014).

[17] L. Du, I. Knez, G. Sullivan, and R. R. Du, Phys. Rev. Lett. 114, 096802 (2015).
[18] V. S. Pribiag, A. J. Beukman, F. Qu, M. C. Cassidy, C. Charpentier, W. Wegscheider, and L. P. Kouwenhoven, Nat. Nanotechnol. 10, 593 (2015).

[19] T. Li, P. Wang, H. Fu, L. Du, K. A. Schreiber, X. Mu, X. Liu, G. Sullivan, G. A. Csathy, X. Lin, and R. R. Du, Phys. Rev. Lett. 115, 136804 (2015).

[20] M. Karalic, S. Mueller, C. Mittag, K. Pakrouski, Q. S. Wu, A. A. Soluyanov, M. Troyer, T. Tschirky, W. Wegscheider, K. Ensslin, and T. Ihn, Phys. Rev. B 94, 241402(R) (2016).

[21] K. H. Kim, D. S. Um, H. Lee, S. Lim, J. Chang, H. C. Koo, M. W. Oh, H. Ko, and H. J. Kim, ACS Nano 7, 9106 (2013).

[22] J. Luo, H. Munekata, F. F. Fang, and P. J. Stiles, Phys. Rev. B 41, 7685 (1990).

[23] G. L. Chen, J. Han, T. T. Huang, S. Datta, and D. B. Janes, Phys. Rev. B 47, 4084 (1993).

[24] J. P. Heida, B. J. van Wees, J. J. Kuipers, T. M. Klapwijk, and G. Borghs, Phys. Rev. B 57, 11911 (1998).

[25] T. Koga, J. Nitta, T. Akazaki, and H. Takayanagi, Phys. Rev. Lett. 89, 046801 (2002).

[26] D. Grundler, Phys. Rev. Lett. 84, 6074 (2000).

[27] C. Schierholz, T. Matsuyama, U. Merkt, and G. Meier, Phys. Rev. B 70, 233311 (2004).

[28] K. H. Kim, H. C. Koo, J. Chang, Y. S. Yang, and H. J. Kim, IEEE Trans. Electron Devices 50, 18 (2014).

[29] C. Brüne, A. Roth, H. Buhmann, E. M. Hankiewicz, L. W. Molenkamp, J. Maciejko, X.-L. Qi, and S.-C. Zhang, Nat. Phys. 8, 486 (2012).

[30] P. D. Dresselhaus, C. M. A. Papavassiliou, R. G. Wheeler, and R. N. Sacks, Phys. Rev. Lett. 68, 106 (1992).

[31] R. J. Elliott, Phys. Rev. 96, 266 (1954).

[32] F. Nichele, M. Kjaergaard, H. J. Suominen, R. Skolasinski, M. Wimmer, B.-M. Nguyen, A. A. Kiselev, W. Yi, M. Sokolich, M. J. Manfra, F. Qu, A. J. A. Beukman, L. P. Kouwenhoven, and C. M. Marcus, Phys. Rev. Lett. 118, 016801 (2017).

[33] S. Hikami, A. I. Larkin, and Y. Nagaoka, Prog. Theor. Phys. 63, 707 (1980)

[34] T. Matsuyama, R. Kürsten, C. Meißner, and U. Merkt, Phys. Rev. B 61, 15588 (2000). 
[35] A. C. H. Rowe, J. Nehls, R. A. Stradling, and R. S. Ferguson, Phys. Rev. B 63, 201307 (2001).

[36] C. Morrison, J. Foronda, P. Wiśniewski, S. D. Rhead, D. R. Leadley, and M. Myronov, Thin Solid Films 602, 84 (2016).

[37] G. Bergmann, Phys. Rep. 107, 1 (1984).

[38] G. Dumpich and A. Carl, Phys. Rev. B 43, 12074 (1991).

[39] Y. H. Park, H.-j. Kim, J. Chang, S. H. Han, J. Eom, H.-J. Choi, and H. C. Koo, Appl. Phys. Lett. 103, 252407 (2013).
[40] K.-H. Kim, H.-j. Kim, H. C. Koo, J. Chang, and S.-H. Han, Appl. Phys. Lett. 97, 012504 (2010).

[41] S. Birner, T. Zibold, T. Andlauer, T. Kubis, M. Sabathil, A. Trellakis, and P. Vogl, IEEE Trans. Electron Devices 54, 2137 (2007).

[42] X.-J. Hao, T. Tu, G. Cao, C. Zhou, H.-O. Li, G.-C. Guo, W. Y. Fung, Z. Ji, G.-P. Guo, and W. Lu, Nano Lett. 10, 2956 (2010).

[43] I. Žutić, J. Fabian, and S. Das Sarma, Rev. Mod. Phys. 76, 323 (2004). 